\title{
Alarming rate of 30-day hospital readmissions in patients with liver cirrhosis
}

\author{
Ebehiwele Ebhohon ${ }^{1}$, Olumuyiwa Akinbolaji Ogundipe ${ }^{2}$, Adeyinka Charles Adejumo ${ }^{3,4}$
}

${ }^{1}$ Department of Internal Medicine, Lincoln Medical Center, Bronx, NY, USA; ${ }^{2}$ Applied Clinical Research Program, St. Cloud State University Minnesota, St. Cloud, MN, USA; ${ }^{3}$ Division of Gastroenterology, Hepatology, and Nutrition, University of Pittsburgh, Pittsburgh, PA, USA; ${ }^{4}$ Individualized Genomics and Health Program, Johns Hopkins University, Baltimore, MD, USA

Correspondence to: Adeyinka Charles Adejumo, MD, MS. Division of Gastroenterology, Hepatology, and Nutrition, University of Pittsburgh, Pittsburgh, PA, USA. Email: adeyinka_adejumo@yahoo.com.

Comment on: Garg SK, Goyal H, Obaitan I, et al. Incidence and predictors of 30-day hospital readmissions for liver cirrhosis: insights from the United States National Readmissions Database. Ann Transl Med 2021;9:1052.

Submitted Oct 02, 2021. Accepted for publication Oct 12, 2021.

doi: $10.21037 /$ atm-21-5258

View this article at: https://dx.doi.org/10.21037/atm-21-5258

Like the terminal stages of heart, kidney, and lung diseases, the risk of morbidity and mortality for patients with advanced liver disease is high. Besides high admission rates, these patients also have disproportionally elevated rates of readmissions. About one-quarter of patients discharged after hospitalization for decompensated liver disease are readmitted within 30 days of hospital discharge $(1,2)$. Consequently, these patients have worse clinical outcomes, a higher 90-day mortality rate, and an increased cost burden to the patients, caregivers, and government. Because readmission rates have been a measure for assessing quality care, there have been significant efforts to identify the etiology of readmissions. Readmissions could be due to several causes, including inevitable complications arising from advanced liver disease, the initial hospital stay, post-discharge follow-up, etc. Regardless of the reason, readmissions impose a massive burden on the healthcare system.

The prevalence and pattern of readmissions have been previously investigated among patients with liver cirrhosis $(3,4)$. Although potential strategies exist to decrease the rates of hospital readmissions in liver disease, they have not translated to a significant decline in the frequency of the condition among patients with liver cirrhosis (5-7). Furthermore, these previous studies among patients with liver disease have been limited to single centers or specific regions within the United States, without any national estimates. Therefore, Garg and Goyal et al. (7) conducted this nationwide study titled "Incidence and Predictors of 30-day bospital readmissions for liver cirrhosis" to provide such highly relevant information in this issue of the Annual of Translational Medicine. The authors used the National Readmissions Database (NRD) from January 2010 to December 2014, a representative sample from 27 geographically diverse states in the United States (7). They analyzed the frequency of new admissions within 30-day of discharge after an index liver cirrhosis related-hospitalization. Only the first episode of readmission was included in the analysis for patients who had more than one readmission within 30-day of discharge. They found an overall 30-day readmissions rate of 31.4\%, which was unchanged from 2010 to 2014. Their multivariate analysis showed that the factors associated with 30-day hospital readmissions for liver cirrhosis included admissions in the weekend, discharges against medical advice, high comorbid burden, and liver-related complications including hepatic encephalopathy, ascites, and non-bleeding esophageal varices. While factors associated with a lower risk of 30-day readmission included female sex, all older age categories (compared to 18-44-year), type of health insurance (private and self-pay compared to Medicare), discharge disposition (discharges to skilled nursing facilities or shortterm hospital compared to discharges to home), having an upper gastrointestinal endoscopy (EGD) during the index hospitalization. Finally, receipt of alcohol rehabilitation during index hospitalization was associated with lower odds of 30-day readmissions.

Many of the findings in this study are consistent with and 
confirm the current literature, as previous studies noted by the authors have reported a 30-day readmission rate of 20$37 \%$ among patients with cirrhosis $(4,8,9)$. Additionally, this article by Garg and Goyal et al. is quite relevant because it provides an insight into the rate and potential predictors of 30-day hospital readmission for liver cirrhosis at a national level. Unfortunately, as acknowledged by the authors, the lack of granularity in the NRD restricted their capability to decipher the reasons underlying most of their findings. First, missing data and misrepresentation, an infamous weakness of administrative claims data, might have impacted the validity of some of their results. For example, the authors did not detect a relationship between median household income and readmissions, contrasting other studies (10-12). Second, the NRD excludes data from institutionalized individuals (such as prisons) or federally funded hospitals (such as Veterans Hospitals) who are known to suffer disproportionately from hepatitis $\mathrm{C}$ and alcohol-related liver diseases, thereby potentially underestimating the rate of readmissions. For instance, Beste et al. revealed that among Veterans, a group not analyzed in this study, the burden of liver cirrhosis is high and increased from 159 to 193 per 100,000 patient-years from 2001 to 2013 (13). Third, the NRD database does not contain outpatient activities, which is known to impact care for patients with cirrhosis significantly. Relevant outpatient information will include (but is not limited to) discharge to alcohol rehabilitation centers, follow-up with an outpatient gastroenterologist, physical therapy, schedule for paracentesis, optimal care during paracentesis (e.g., receipt of albumin replacement), home health nurse visitations, etc. $(14,15)$. Fourth, since patients analyzed in this study can only be linked to one state, those with readmissions after an index hospitalization for liver cirrhosis and its related complications in another state are frequently lost to follow-up, which could further impact the accuracy of the nationwide estimates. Fifth, the NRD database does not contain medications such as diuretics, rifaximin, lactulose, acamprosate, disulfiram, etc., or compliance to dietary or lifestyle modifications, all of which impacts care. Furthermore, the authors aggregated the etiology of liver cirrhosis into three exclusive groups (alcoholic, biliary and non-alcoholic). The non-alcoholic group was a large heterogeneous collection of different liver diseases including viral hepatitides, hemochromatosis, etc. which all may behave differently. It will be exciting to see the differences in pattern of readmission of these different etiologies of cirrhosis in subsequent studies. For example, patients with NASH may be investigated for higher readmissions for cardiovascular conditions. Finally, patients who have an endoscopy had a lower risk of 30-day readmissions, suggesting that offering inpatient endoscopy to patients admitted for liver cirrhosis may be beneficial. However, without details (limitation of NRD) of the indications for the index admission and for the endoscopy, concerns for gastrointestinal bleeding and the provision of outpatient endoscopy among the groups who did not receive endoscopy, the application of this information is limited.

Several studies have shown that patient readmissions are often associated with liver cirrhosis-related complications and the presence of other comorbidities. Therefore, an indepth analysis of hospital readmissions for liver cirrhosis in the United States with more granular data is necessary to a variety of stakeholders who view readmission as a quality-of-care measure for improved survival in these patients. Although complications of cirrhosis such as hepatic encephalopathy, variceal bleeding, and infection can be unavoidable and indicate the need for high-quality care, some predictors of re-hospitalizations are potentially avoidable. As suggested by Garg and Goyal et al., factors that influence readmissions, such as patient education and social support, need to be assessed in future studies to help improve the survival rate for patients with liver cirrhosis (7). Other promising potential strategies based on existing and current data emphasize the implementation of optimal hospital post-discharge care to reduce livercirrhosis-related complications, leading to a reduction in the number of readmitted patients within 30 days of hospital discharge (15). In the meantime, investigators seeking to find effective ways to mitigate 30 days readmissions for liver cirrhosis should prioritize prospective studies with more granular data that will help determine the mechanism underlying the predictors associated with high rates of 30-day readmissions identified in this study by Garg and Goyal et al. Unfortunately, even if subsequent studies identify the reasons enabling these factors related to 30 day readmissions, and if recommendations are provided to mitigate these factors, most of them are often not adopted. Therefore, only widespread adoption of implementation science to hepatology would genuinely enable the execution of any evidence-based practice to decrease the burden of readmissions among patients with advanced liver disease (16).

\section{Acknowledgments}

Funding: This work was partially supported by an NIH T32DK063922 grant. The funders had no role in any of the 
opinions in this manuscript.

\section{Footnote}

Provenance and Peer Review: This article was commissioned by the editorial office, Annals of Translational Medicine. The article did not undergo external peer review.

Conflicts of Interest: All authors have completed the ICMJE uniform disclosure form (available at https://dx.doi. org/10.21037/atm-21-5258). The authors have no conflicts of interest to declare.

Ethical Statement: The authors are accountable for all aspects of the work in ensuring that questions related to the accuracy or integrity of any part of the work are appropriately investigated and resolved.

Open Access Statement: This is an Open Access article distributed in accordance with the Creative Commons Attribution-NonCommercial-NoDerivs 4.0 International License (CC BY-NC-ND 4.0), which permits the noncommercial replication and distribution of the article with the strict proviso that no changes or edits are made and the original work is properly cited (including links to both the formal publication through the relevant DOI and the license). See: https://creativecommons.org/licenses/by-nc-nd/4.0/.

\section{References}

1. Tapper EB, Finkelstein D, Mittleman MA, et al. Standard assessments of frailty are validated predictors of mortality in hospitalized patients with cirrhosis. Hepatology 2015;62:584-90.

2. Tapper EB, Volk M. Strategies to Reduce 30Day Readmissions in Patients with Cirrhosis. Curr Gastroenterol Rep 2017;19:1.

3. Scaglione S, Kliethermes S, Cao G, et al. The Epidemiology of Cirrhosis in the United States: A Population-based Study. J Clin Gastroenterol 2015;49:690-6.

4. Berman K, Tandra S, Forssell K, et al. Incidence and predictors of 30-day readmission among patients hospitalized for advanced liver disease. Clin Gastroenterol Hepatol 2011;9:254-9.

5. Agrawal K, Kumar P, Markert R, et al. Risk Factors for 30-Day Readmissions of Individuals with Decompensated Cirrhosis. South Med J 2015;108:682-7.

6. Weinreich MA, Styrvoky K, Chang S, et al. Sepsis at a
Safety Net Hospital: Risk Factors Associated With 30-Day Readmission. J Intensive Care Med 2019;34:1017-22.

7. Garg SK, Goyal H, Obaitan I, et al. Incidence and predictors of 30-day hospital readmissions for liver cirrhosis: insights from the United States National Readmissions Database. Ann Transl Med 2021;9:1052.

8. Adejumo AC, Cholankeril G, Iqbal U, et al. Readmission Rates and Associated Outcomes for Alcoholic Hepatitis: A Nationwide Cohort Study. Dig Dis Sci 2020;65:9901002.

9. Volk ML, Tocco RS, Bazick J, et al. Hospital readmissions among patients with decompensated cirrhosis. Am J Gastroenterol 2012;107:247-52.

10. Brahmania M, Wiskar K, Walley KR, et al. Lower household income is associated with an increased risk of hospital readmission in patients with decompensated cirrhosis. J Gastroenterol Hepatol 2021;36:1088-94.

11. Patil S, Shah M, Patel B, et al. Readmissions Among Patients Admitted With Acute Decompensated Heart Failure Based on Income Quartiles. Mayo Clin Proc 2019;94:1939-50.

12. Brown CS, Montgomery JR, Neiman PU, et al. Assessment of Potentially Preventable Hospital Readmissions After Major Surgery and Association With Public vs Private Health Insurance and Comorbidities. JAMA Netw Open 2021;4:e215503.

13. Beste LA, Leipertz SL, Green PK, et al. Trends in burden of cirrhosis and hepatocellular carcinoma by underlying liver disease in US veterans, 2001-2013. Gastroenterology 2015;149:1471-1482.e5; quiz e17-8.

14. Kanwal F, Asch SM, Kramer JR, et al. Early outpatient follow-up and 30-day outcomes in patients hospitalized with cirrhosis.Hepatology 2016;64:569-81.

15. Morales BP, Planas R, Bartoli R, et al. HEPACONTROL. A program that reduces early readmissions, mortality at 60 days, and healthcare costs in decompensated cirrhosis. Dig Liver Dis 2018;50:76-83.

16. Rogal SS, Powell BJ, Chinman M, et al. Moving Toward Impact: An Introduction to Implementation Science for Gastroenterologists and Hepatologists. Gastroenterology 2020;159:2007-12.

Cite this article as: Ebhohon E, Ogundipe OA, Adejumo AC. Alarming rate of 30-day hospital readmissions in patients with liver cirrhosis. Ann Transl Med 2021;9(21):1608. doi: 10.21037/ atm-21-5258 